\title{
DC Characterisation of C60 Whiskers and Nanowhiskers
}

\author{
Larsson, Michael; Kjelstrup-Hansen, Jakob; Lucyszyn, Stepan
}

Published in:

E C S Transactions

Link to article, DOI:

$10.1149 / 1.2408950$

Publication date:

2007

Document Version

Publisher's PDF, also known as Version of record

Link back to DTU Orbit

Citation (APA):

Larsson, M., Kjelstrup-Hansen, J., \& Lucyszyn, S. (2007). DC Characterisation of C60 Whiskers and Nanowhiskers. E C S Transactions, 2(12), 27-38. https://doi.org/10.1149/1.2408950

\section{General rights}

Copyright and moral rights for the publications made accessible in the public portal are retained by the authors and/or other copyright owners and it is a condition of accessing publications that users recognise and abide by the legal requirements associated with these rights.

- Users may download and print one copy of any publication from the public portal for the purpose of private study or research.

- You may not further distribute the material or use it for any profit-making activity or commercial gain

- You may freely distribute the URL identifying the publication in the public portal

If you believe that this document breaches copyright please contact us providing details, and we will remove access to the work immediately and investigate your claim. 


\title{
DC Characterisation of $\mathbf{C}_{60}$ Whiskers and Nanowhiskers
}

\author{
M. P. Larsson ${ }^{\mathrm{a}}$, J. Kjelstrup-Hansen ${ }^{\mathrm{b}}$ and S. Lucyszyn ${ }^{\mathrm{a}}$ \\ ${ }^{a}$ Department of Electrical \& Electronic Engineering, Imperial College, London, UK \\ ${ }^{b}$ Department of Micro and Nanotechnology, Technical University of Denmark, Lyngby, \\ Denmark
}

$\mathrm{C}_{60}$ whiskers exhibit increasing conductivity with decreasing diameter. At diameters of $1 \mu \mathrm{m}$ and below, a single-crystal structure predominates, and enhanced electrical characteristics are expected; however, no supporting data exists in the literature. Here, results of four-point probe measurements on $\mathrm{C}_{60}$ whiskers and nanowhiskers with diameters in the range $650 \mathrm{~nm}$ to $1.3 \mu \mathrm{m}$ are reported for the first time. Samples are attached to pre-patterned planar and raised electrodes using FIB-deposited tungsten. A low resistivity of $3 \Omega \mathrm{cm}$ is measured in air, on a $\mathrm{C}_{60}$ whisker having a diameter of $650 \mathrm{~nm}$, indicating strong potential for use in organic electronic applications of the future. Repeated current cycling in air is observed to promote sample degradation, possibly due to progressive oxidation of the carbon structure. A micromachined four-point probe is also used to try to establish non-invasive electrical connections with samples. Preliminary results of such trials are presented, indicating it to be a feasible alternative to the use of deposited electrodes.

\section{Introduction}

Since the discovery of fullerene $\mathrm{C}_{60}$, by Kroto et al. [1], advances in the field of fullerene chemistry have led to a number of interesting developments, such as the carbon nanotube (CNT) [2]. The $\mathrm{C}_{60}$ molecule consists of carbon atoms located at the nodes of a series of hexagons and pentagons arranged in a cage lattice, defined by alternating double and single bonds. The double bonds are not energetically stable within pentagon rings, giving the $\mathrm{C}_{60}$ molecule an overall electron-withdrawing character. The curvature induced by the cage structure increases the energy associated with the double bonds, further enhancing the reactivity of the molecule. It is for these reasons that $\mathrm{C}_{60}$ molecules are susceptible to nucleophilic addition as well as cycloaddition reactions.

$\mathrm{C}_{60}$ molecules are known to polymerise under high temperature, high pressure conditions [3] and in the presence of ultraviolet (UV) radiation [4]. In each case, the mechanism driving polymerisation is suggested to be $2+2$ cycloaddition. This involves the breaking of parallel double bonds on adjacent $\mathrm{C}_{60}$ molecules and their reformation into four-membered, cross-linking rings. Such bonding can occur to varying degrees, giving rise to polymerised $\mathrm{C}_{60}$ structures with cross-linking bonds in 1-, 2- and 3-dimensions. The reduction in the number of double bonds following $2+2$ cycloaddition reduces the strain energy in the $\mathrm{C}_{60}$ structure, allowing molecules to elongate in directions parallel to the new cross-links. The replacement of intermolecular Van der Waals bonds covalent C-C bonds, increases the strain associated with the polymer structure over that of pristine $\mathrm{C}_{60}$ crystals.

Recently, Miyazawa et al. [5] reported technique for growing needle-like structures of $\mathrm{C}_{60}$ from solution at room temperature. The needles grew in arbitrary directions through a 
process of liquid-liquid interfacial precipitation (LLIP), between $\mathrm{C}_{60}$ saturated toluene and Isopropyl alcohol (IPA); Figure 1a. Diameters ranged from hundreds of nanometres to several microns, with lengths reaching several hundreds of microns. Needles with diameters below $1 \mu \mathrm{m}$ were referred to as $\mathrm{C}_{60}$ nanowhiskers, and those with larger diameters were described as $\mathrm{C}_{60}$ whiskers (Figure $1 \mathrm{~b}$ ).

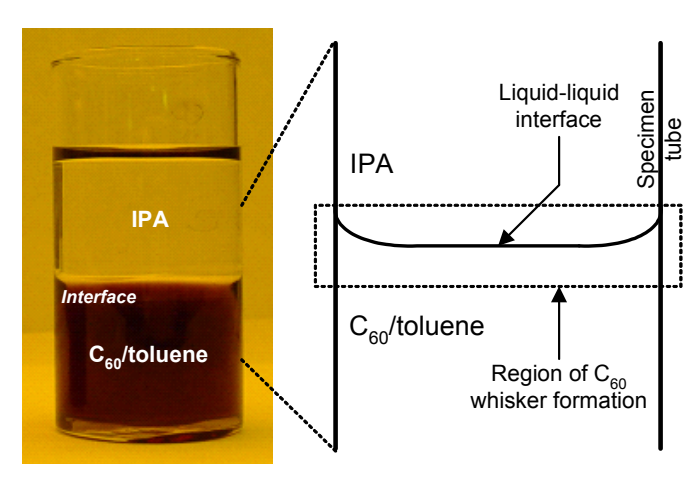

a)

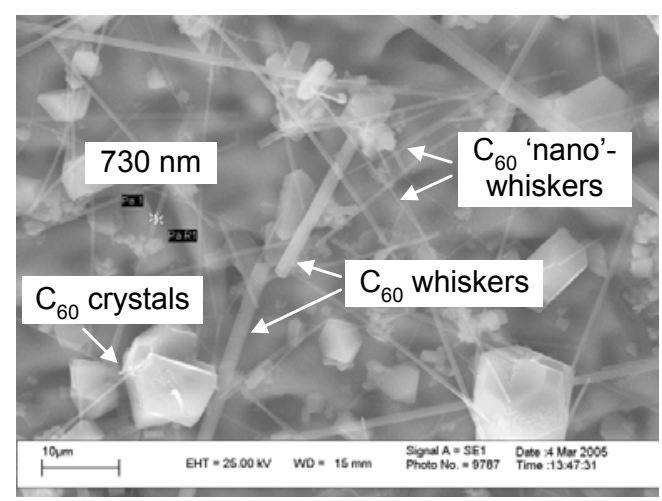

b)

Figure 1. a) Liquid-liquid interface between IPA and $\mathrm{C}_{60} /$ toluene: a) growth vial indicating region in which $\mathrm{C}_{60}$ whiskers are observed to grow; and b) products found within the liquid-liquid interface following several hours of growth.

In contrast to CNTs, which can be visualised as rolled-up sheets of graphene, $\mathrm{C}_{60}$ whiskers contain no long-range hollow structure. Instead, structural characterisation $[5,6]$ has indicated the whiskers are solid, consisting of a series of $\mathrm{C}_{60}$ molecules, bounded through a combination of Van der Waals interactions and chemical bonds. The latter suggests that $\mathrm{C}_{60}$ whiskers contain chains of polymerised $\mathrm{C}_{60}$ within their structure.

A transition from poly- to single-crystalline structure is seen when the diameter of $\mathrm{C}_{60}$ whiskers decreases to $1 \mu \mathrm{m}$ and below. The absence of grain boundaries, combined with the observation of enhanced intermolecular bonding, are the main reasons why $\mathrm{C}_{60}$ nanowhiskers are expected to exhibit superior electrical and mechanical properties to those of their larger-diameter counterparts.

Two-point probe (2PP) measurements on $\mathrm{C}_{60}$ whiskers, suspended between indiumgallium electrodes, at room temperature reveal a proportional relationship between whisker diameter and DC resistivity [6] (solid line, Figure 2). $\mathrm{C}_{60}$ whiskers measuring less than $10 \mu \mathrm{m}$ in diameter were observed to posses lower resistivities compared to that of bulk $\mathrm{C}_{60}$ crystal. A minimum value of $10^{5} \Omega \mathrm{cm}$ was reported for a $\mathrm{C}_{60}$ whisker having a diameter of approximately $7 \mu \mathrm{m}$. This value includes contact resistance between the sample and the measurement electrodes, so it is not a true indicator of the intrinsic electrical characteristics of the sample.

Despite the expectation of considerably lower resistivity in $\mathrm{C}_{60}$ whiskers with diameters approaching $1 \mu \mathrm{m}$ and below (dotted line, Figure 2), measurements to confirm this assertion are not reported in the literature. If correct, the combination of desirable electrical and mechanical attributes may make $\mathrm{C}_{60}$ nanowhiskers suitable candidates for applications in organic-based electronics, for devices such as RF power sensors [7] or field-effect transistors [8].

This paper, for the first time, presents results of DC measurements on individual $\mathrm{C}_{60}$ whiskers with diameters close to $1 \mu \mathrm{m}$ and two $\mathrm{C}_{60}$ nanowhiskers with sub-micron diameters. In all cases, the samples are permanently attached to the measurement 
electrodes using focused ion beam (FIB) -assisted deposition of metal. To offer a less invasive route towards electrical characterisation, details of preliminary work into the use of a micromachined, contacting four-point probe ( $\mu-4 \mathrm{PP})$ are presented. Measurements with this system are, however, limited to larger-diameter $\mathrm{C}_{60}$ whiskers as will be discussed, so direct comparison with the former set of data is not possible.

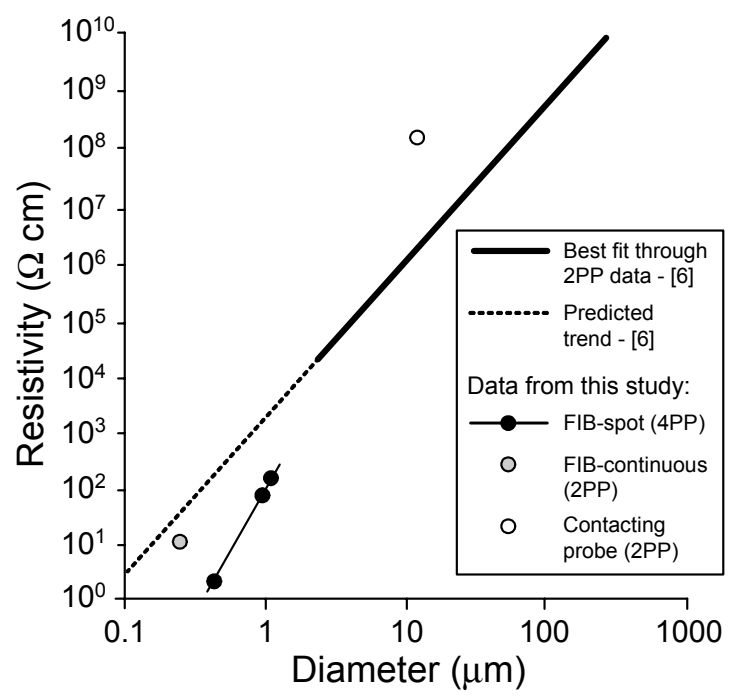

Figure 2. $\quad$ DC resistivity of $\mathrm{C}_{60}$ whiskers with diameter: combined plot showing data from the literature [6], including new 2- and 4PP data obtained using deposited and contacting electrodes.

\section{Experimental}

$\mathrm{C}_{60}$ nanowhiskers were grown via liquid-liquid precipitation, adopting a procedure similar to that described in [5]. The samples were then applied onto thermally oxidised $\mathrm{Si}$ substrates for electrical characterisation. Some of the substrates contained planar or raised pre-patterned metal tracks, to reduce the level of FIB processing necessary to establish electrical connections to the samples. Electrical measurements were also attempted using a commercial $\mu-4 \mathrm{PP}$ to evaluate the relative merits of using contacting rather than permanent (deposited) electrodes. The following sections provide details on $\mathrm{C}_{60}$ whisker (and nanowhisker) growth, substrate preparation and means of characterisation.

\section{Whisker growth}

Growth solutions were prepared in $25 \mathrm{ml}$ glass vials. First, $\mathrm{C}_{60}$ powder with a purity of 99+\% (M.E.R. Corp., US) was sonically dissolved in toluene to achieve a saturated solution of between $0.2-0.3 \%$ concentration by weight. The $\mathrm{C}_{60} /$ toluene solution was then transferred to separate vials in volumes of $8 \mathrm{ml}$, to which equal measures of isopropyl alcohol (IPA) were gently added. Differences in surface energy between the respective liquids led to the formation of a liquid-liquid interface as soon as the IPA was added (Figure 1a). Growth of $\mathrm{C}_{60}$ whiskers was observed to occur in the proximity of this region.

$\mathrm{C}_{60}$ whisker growth follows a period of nucleation, during which $\mathrm{C}_{60}$ crystals form on surfaces in the vicinity of the liquid-liquid interface. $\mathrm{C}_{60}$ whiskers are observed to grow in arbitrary directions from these nucleation sites, reaching lengths of several hundreds of microns after only a few hours. Whisker diameters are typically in the range of several hundred microns to several microns. Prolonged growth periods lead to an increase in average whisker dimensions. As such, it is likely that whiskers initially grow as single, molecule-wide strands (Figure 3), gradually increasing in diameter as similar strands come 
within close proximity and pack together through intermolecular interactions. The larger intermolecular separation in directions perpendicular to the growth axis [5] confirms bonding is not as robust as that along the growth axis. Aside from whiskers, crystals (Figure 1b) and spherical clusters (Figure 4) of $\mathrm{C}_{60}$ also precipitated at the liquid-liquid interface. When replaced in fresh toluene solution, newly-grown $\mathrm{C}_{60}$ whiskers maintained their structural integrity. This indicated the presence of enhanced intermolecular bonding, compared with that found in pristine $\mathrm{C}_{60}$ crystals, supporting the suggestion that $\mathrm{C}_{60}$ whiskers contain polymerised chains of $\mathrm{C}_{60}$. Sonic agitation did, however, induce structural damage, breaking the whiskers into shorter lengths.

Glass capillary tubes were placed through the liquid-liquid interface in some vials, to provide additional surface area onto which $\mathrm{C}_{60}$ whiskers could grow. Although exposure to ultra-violet radiation can enhance polymerisation between $\mathrm{C}_{60}$ molecules, it is not a prerequisite in the LLIP technique. Vials were thus sealed with polypropylene caps and placed within an opaque container, for a growth period lasting a total of 72 hours. following the growth period, precipitated $\mathrm{C}_{60}$ whiskers were collected and dispersed into larger vials, containing solutions of either ethanol or IPA, for storage. The samples were then applied to various thermally oxidised $\mathrm{Si}$ substrates using a glass pipette.

\section{Possible growth mechanism}

The expected mechanism underlying the growth of $\mathrm{C}_{60}$ whiskers and nanowhiskers is still uncertain. Observations of reduced intermolecular spacing along the growth direction of $\mathrm{C}_{60}$ nanowhiskers, below that found in bulk $\mathrm{C}_{60}$ crystals, indicated the presence of covalent cross-linking bonds. This prompted speculation that polymerisation had occurred between $\mathrm{C}_{60}$ molecules via $2+2$ cycloaddition, leading to the formation of a series of 1dimensional (1D) "pearl-chain" structures [5]. Differences in surface energy between IPA and $\mathrm{C}_{60}$ /toluene generate a pressure gradient across the interface, which varies inversely with its radius of curvature. The pressure within the liquid-liquid interface region, is thus considered to be large enough to induce cycloaddition reactions at room temperature. This possibility can certainly not be ruled out, as $1 \mathrm{D}$ chains of polymerised $\mathrm{C}_{60}$ are known to form under high pressure conditions, without the need for high temperatures [9]. Lateral bonding between individual chains is assumed to be through Van der Waals interactions, as is the case in bulk $\mathrm{C}_{60}$ crystals, as no indication of chemical bonding was observed in other directions. Figure 3 indicates the manner in which $\mathrm{C}_{60}$ molecules polymerise via $2+2$ cycloaddition, leading to the formation of a dimer and a 1D chain.
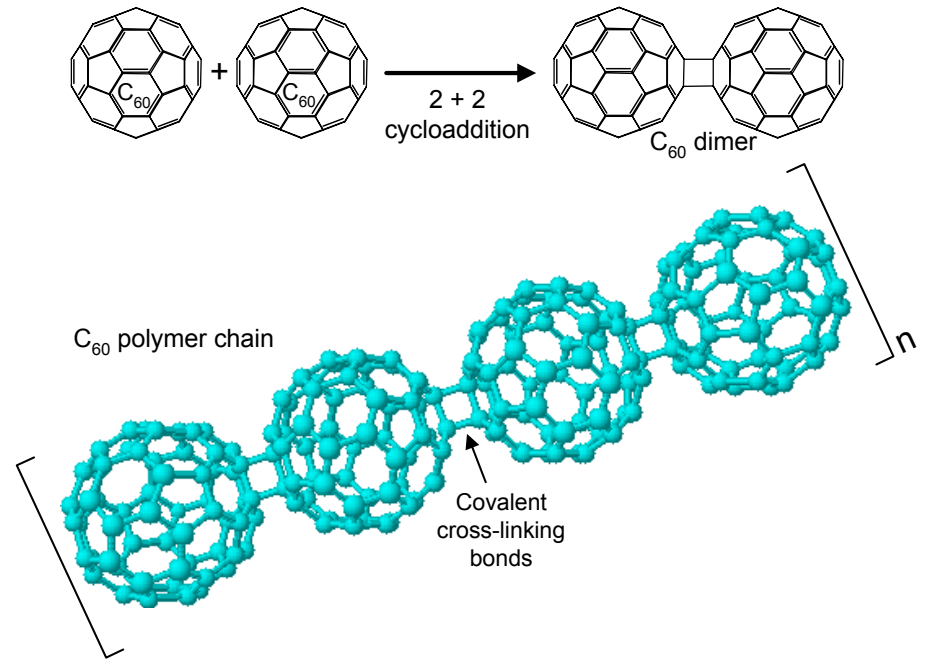

Figure 3. Polymerisation between adjacent $\mathrm{C}_{60}$ molecules: a possible mechanism for the growth of $\mathrm{C}_{60}$ nanowhiskers via LLIP. 


\section{$\underline{\text { Substrate preparation }}$}

Standard 4" Si wafers were thermally oxidised at $1100{ }^{\circ} \mathrm{C}$ to achieve a silica $\left(\mathrm{SiO}_{2}\right)$ layer thickness of $200 \mathrm{~nm}$. The substrates were then divided into three lots. Two lots (A and $\mathrm{B}$ ) received further processing to form wither planar or raised electrodes, whilst the third lot (C) remained unprocessed. Sputtered layers of chrome $(\mathrm{Cr})$ and tungsten (W) were sequentially applied to thicknesses of $500 \AA$ and $1000 \AA$, respectively, on substrates in lots $\mathrm{A}$ and $\mathrm{B}$. The bimetallic layer was then patterned to define $2-\mu \mathrm{m}$ wide strips, and larger $\left(500 \times 500 \mu^{2}\right)$ connecting pads, by wet etching using a $1.4 \mu \mathrm{m}$ thick, hard-baked photoresist mask, formed with S1828 (Shipley, UK). The tungsten layer was removed following immersion in a 1:1 solution of buffered hydrofluoric acid (BHF) and nitric acid $\left(\mathrm{HNO}_{3}\right)$ for a period of 15 seconds. The photoresist layer was retained to allow patterning of the underlying $\mathrm{Cr}$ layer in a 1:5:25 aqueous solution containing ceric ammonium nitrate, nitric acid and water.

Substrates in lot A were stripped of their photoresist mask, and a period of oxygen $\left(\mathrm{O}_{2}\right)$ plasma treatment followed to ensure the removal of organic remnants. The patterned photoresist was retained on substrates in lot $\mathrm{B}$ and dehydrated in an oven set to $120{ }^{\circ} \mathrm{C}$ for 30 minutes, to remove water absorbed during wet etching. The $\mathrm{SiO}_{2}$ layer between each electrode was then removed through reactive ion etching (RIE), revealing the underlying $\mathrm{Si}$ substrate. DRIE processing followed, to etch regions of exposed Si to depths in the range 5-10 $\mu \mathrm{m}$, leaving 'raised' electrodes. Removal of the photoresist mask then followed, in the manner described above.

All the substrates were then cleaved into 1 x $1 \mathrm{~cm}^{2}$ dies, onto which $\mathrm{C}_{60}$ whiskers were applied, by slowly adding solutions (IPA or ethanol) in which they were dispersed, dropwise, using a glass pipette.

\section{FIB-deposited electrodes}

Fixed, ohmic electrical connections were made to $\mathrm{C}_{60}$ whiskers using FIB-deposited metal. For samples placed on substrate dies from lot C, FIB-deposition was required to define measurement electrodes and contact pads to enable probing in a conventional DC probestation. Figure $4 \mathrm{a}$ shows tracks and pads formed in platinum $(\mathrm{Pt})$ on a $\mathrm{C}_{60}$ nanowhisker having a diameter of $400 \mathrm{~nm}$. The FIB process employed a beam of gallium (Ga) ions, generated at $30 \mathrm{keV}$ and between $2-6 \mathrm{pA} / \mathrm{cm}^{2}$.

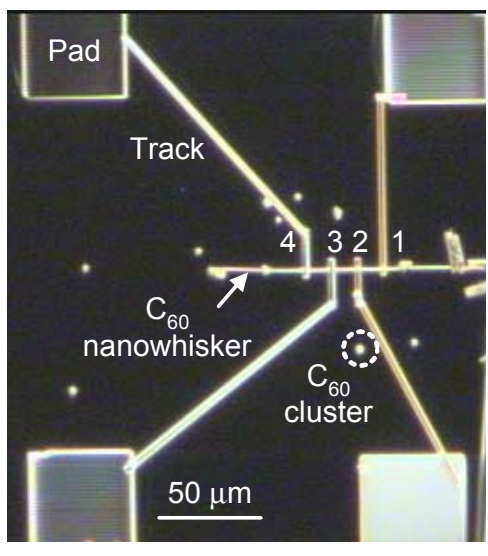

a)

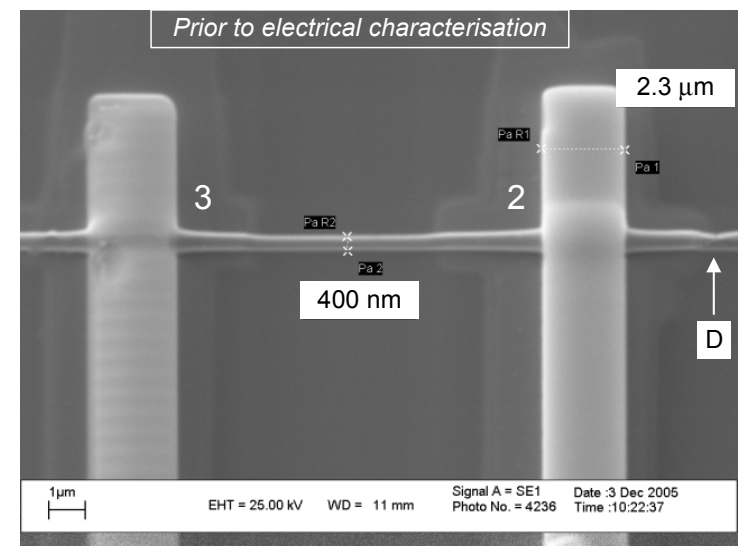

b)

Figure 4. FIB-deposited Pt tracks and pads to enable electrical characterisation of a $400 \mathrm{~nm}$ diameter $\mathrm{C}_{60}$ nanowhisker: a) microscope image showing sample, tracks and pads; and b) close-up of section between the inner two electrodes. 
Electrodes were deposited to a thickness and a width of $0.5 \mu \mathrm{m}$ and $2 \mu \mathrm{m}$, respectively, intersecting the nanowhisker at equidistant locations, spaced at $10 \mu \mathrm{m}$. Each electrode was connected to a larger pad, measuring $50 \times 50 \mu \mathrm{m}$, to establish contact with conventional probe tips (Figure 4a).

Initial electrical measurements revealed the presence of a thin, residual conducting film in regions between the newly deposited electrodes. This film could be removed in selected areas through FIB etching using a beam of $\mathrm{Ga}$ ions, isolating adjacent electrodes to the nanowhisker. Figure $4 \mathrm{~b}$ shows a close-up of the nanowhisker, contacted between the inner electrode pair of the set. Remnants of residual Pt surrounding each electrode are just visible following FIB etching. Pads were contacted using stainless steel probe needles, having a tip radius of $5 \mu \mathrm{m}$, and 2PP current-voltage (I-V) data were extracted between various electrode pairs using a Hewlett Packard 4155B Parameter Analyser.

The level of FIB-processing was significantly reduced in the case of $\mathrm{C}_{60}$ whiskers applied to dies from lots $\mathrm{A}$ and $\mathrm{B}$, due to the presence of pre-patterned electrodes and probing pads. Metal deposition was only required in small regions at intersections between the whiskers and underlying electrodes, to fix the samples in place, and establish electrical contact. In this case, deposits were formed in $\mathrm{W}$, to coincide with the contact material present in the pre-patterned electrodes. W was chosen over gold $(\mathrm{Au})$ and $\mathrm{Pt}$, out of convenience, due to the absence of i) a Pt target and ii) a Au source, for sputter-deposition and FIB-deposition, respectively. $\mathrm{C}_{60}$ whiskers fixed to pre-patterned planar and raised electrodes, following FIB-deposition of W, are shown in Figure 5; in each case the sample diameter is over $1 \mu \mathrm{m}$.

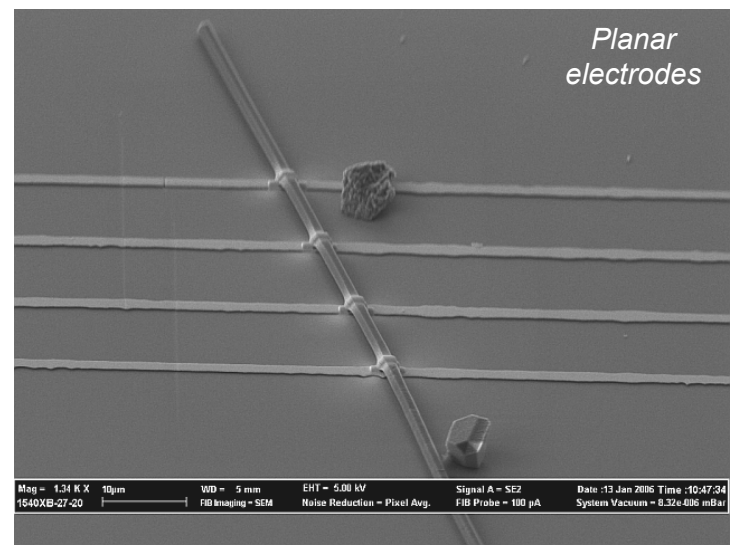

a)

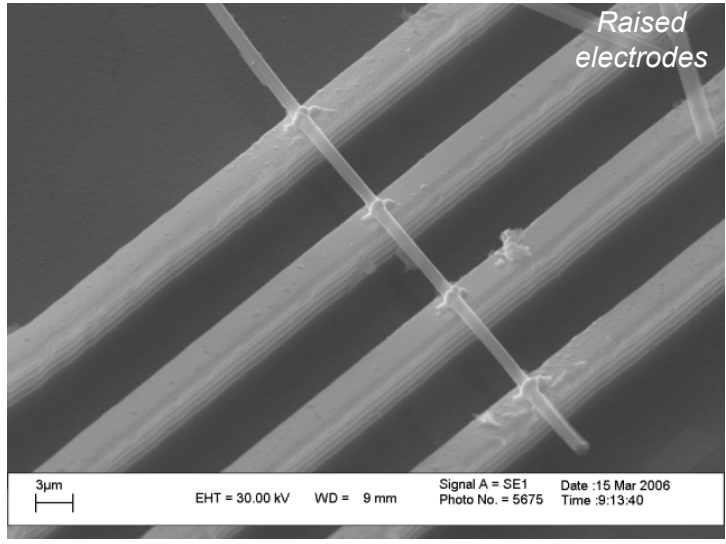

b)

Figure 5. $\quad \mathrm{C}_{60}$ whiskers attached to pre-patterned a) planar; and b) raised electrodes by selective FIB-deposition of W.

Measurements on reference electrodes containing FIB-deposited W confirmed electrical isolation between all sets of electrodes. This was true even for dies containing raised electrodes, despite the presence of exposed $\mathrm{Si}$ in their sidewalls. It is likely that remnant metal deposition, outside defined regions of interest, were insufficiently thick to form a continuous layer, as needed to provide an electrical path between the electrodes and the Si substrate. 


\section{Micromachined four-point probe $(\mu-4 \mathrm{PP})$}

The use of FIB-deposition to form fixed electrical contacts with $\mathrm{C}_{60}$ whiskers is invasive, even when only forming spot-contacts between the samples and the underlying electrodes. Residual metal deposition around intentionally-defined regions can lower the measured resistivity of the sample, whereas implantation of $\mathrm{Ga}$ ions will cause damage to the lattice structure, leading to an increase in resistivity. Conveniently, these separate effects act in opposition, however, their net effect on measured I-V characteristics is uncertain.

An alternative means of establishing electrical contact with $\mathrm{C}_{60}$ nanowhiskers was explored, using a micromachined four-point probe and measurement system [10], developed at the Technical University of Denmark and commercialised through Capres A/S. The probe contains compliant Au-coated silica cantilevers, supported on a Si die, and contact is made with samples using a high-resolution $x-y-z$ positioning stage. Differences in levelling between sample and probe made it difficult to establish electrical contact with all four electrodes on the narrowest $\mathrm{C}_{60}$ whiskers. When the contact pressure was increased to compensate, this often resulted in sample fracture. As such, electrical measurements were limited to whiskers with diameters above $5 \mu \mathrm{m}$. All characterisation was undertaken with nitrogen $\left(\mathrm{N}_{2}\right)$ passed across the sample, as this was found to reduce the inter-electrode leakage current.

\section{Results and Discussion}

\section{Deposited electrodes}

2PP measurements were conducted between pairs of electrodes in the sample shown in Figure 4. The results revealed a wide variation in resistivity, possibly reflecting varying levels of damage sustained during FIB-deposition and subsequent etching. Figure 6a shows I-V characteristics measured along various sections of the $\mathrm{C}_{60}$ nanowhisker. Highest currents were passed through the length between the inner electrodes (electrodes 2-3), yielding a resistivity of $17 \Omega \mathrm{cm}$. Measurements between other electrode combinations (particularly pair 1-2) revealed higher resistivities, indicative of sample damage, sustained during FIB processing. Evidence to support this comes in the form of a thinned section in the nanowhisker, adjacent to electrode 2 (point D, Figure 4b).

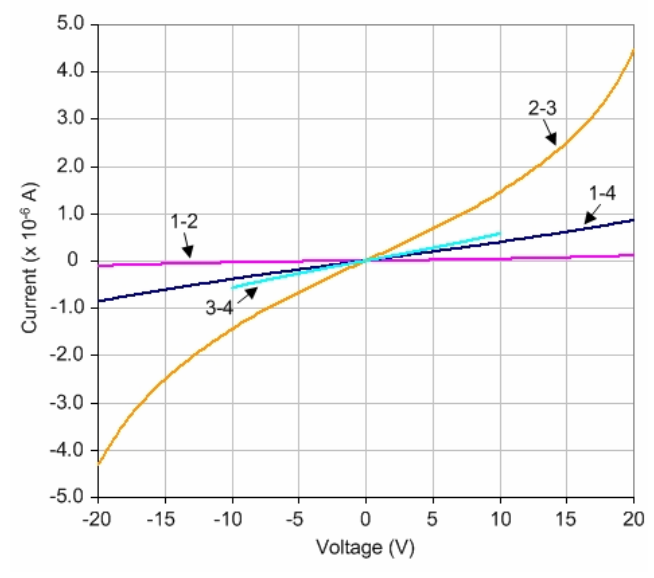

a)

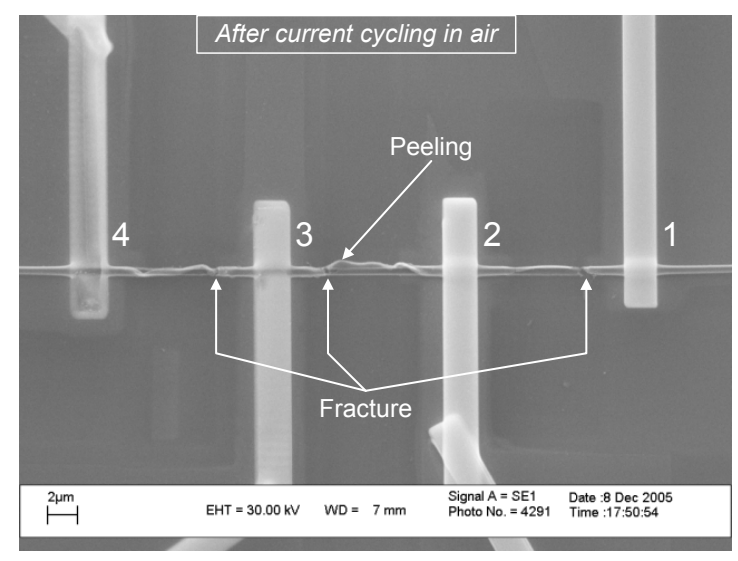

b)

Figure 6. DC characterisation of a $\mathrm{C}_{60}$ nanowhisker in air: a) I-V characteristics between various electrode pairs; and b) sample damage sustained during testing. 
The implantation of Ga ions into the nanowhisker structure, during FIB processing, is another factor that may explain the variability in I-V data. As the sample was not annealed, however, p-type doping is unlikely to have occurred. Instead, changes to electrical resistivity will arise, from disruptions to the periodicity of the single-crystal structure. This will have the effect of reducing carrier mobility, leading to an increase in sample resistivity.

Successive measurements on the sample led to degradation and sample fracture in several locations. Unfortunately, 4PP data were not obtained prior to the first occurrence of sample fracture. Figure $6 \mathrm{~b}$ shows the state of the sample after several sweeps of current between electrode pairs. Signs of sample fracture are visible between each electrode pair and peeling is observed along the majority of the sample's length. As measurements were conducted in air, a possible explanation for the observed degradation is progressive oxidation. This can arise following resistive heating of the sample with applied current. Sample heating can give rise to another degradation mechanism, based on thermallyinduced straining. Variations in the nature of bonding along different directions of the structure can create such a situation. The detrimental effect of intensive FIB processing cannot be ruled out as a contributory factor in both situations and this is likely, at the very least, to have accelerated the degradation process.

A number of 4PP measurements were conducted on $\mathrm{C}_{60}$ whiskers with diameters close to $1 \mu \mathrm{m}$, attached to pre-patterned, planar electrodes. Despite similarities in whisker diameter, and in FIB processing conditions, the results reveal quite a significant variation in I-V characteristics (Figure 7). As before, variations in the extent of sample-damage sustained during FIB processing is a possible explanation. A second possibility is due to differences in charge accumulation within regions of the $\mathrm{SiO}_{2}$ substrate layer in contact with overlying samples. Such charges can interfere with the normal passage of current through the samples, resulting in significant deviations from their intrinsic I-V behaviour. This latter possibility was foreseen, explaining the decision to fabricate substrate dies with raised electrodes.

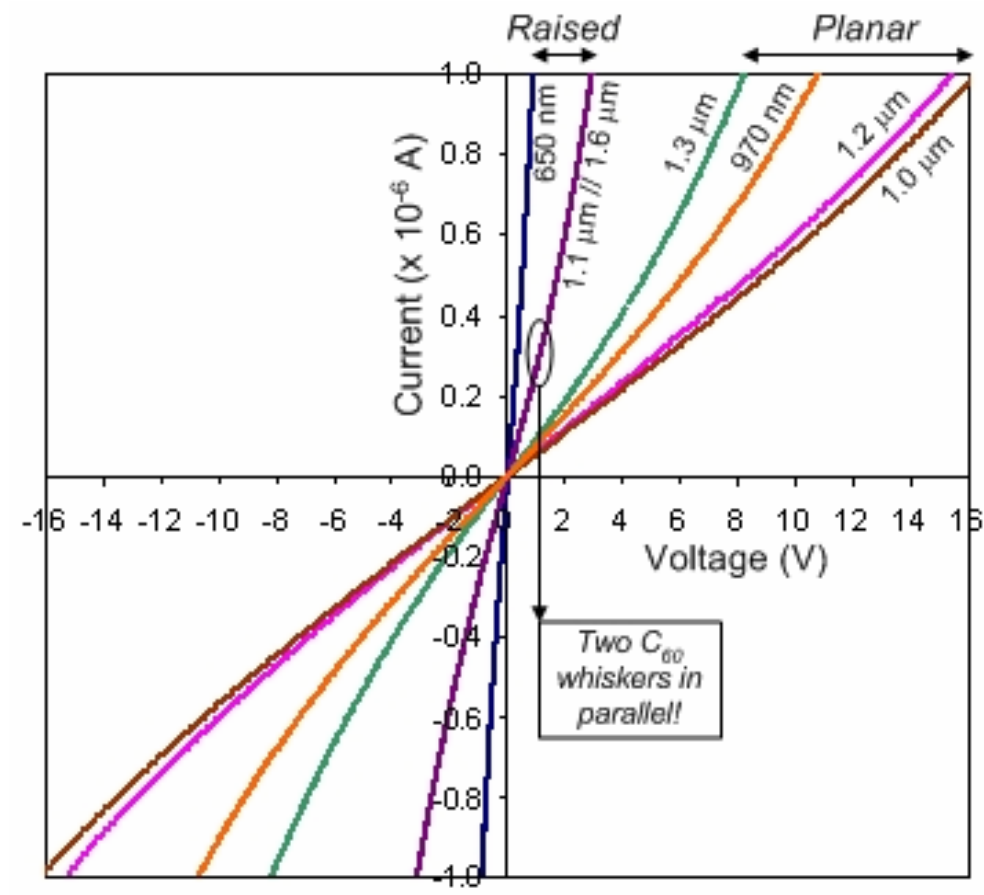

Figure 7. $\quad \mathrm{I}-\mathrm{V}$ measurements from $\mathrm{C}_{60}$ whiskers and nanowhiskers attached, via FIBdeposition, to planar and raised electrodes. 
As a limited number of samples were successfully applied, and then attached, to raised electrodes, the effect of inter-electrode substrate removal could not be investigated. Only two such samples were fabricated, one containing a nanowhisker having a diameter of $650 \mathrm{~nm}$, and the other containing two whiskers of diameter 1.1 and $1.6 \mu \mathrm{m}$, respectively, attached across a common set of electrodes. The latter effectively functioned as a parallel combination of whiskers, yielding resistivity data approximately half that for individual $\mathrm{C}_{60}$ whiskers of similar average diameter. In all cases shown in Figure 7 , successive sweeps of current let to progressive sample damage and eventual fracture. Figure 8 shows a sample attached to raised electrodes before and after electrical characterisation. Damage is less extensive than that in Figure 6b, and localised to a region between the inner pair of electrodes.

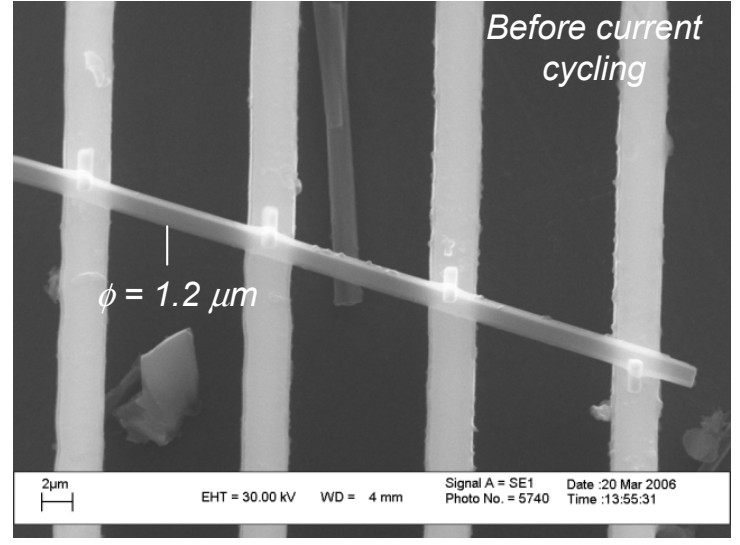

a)

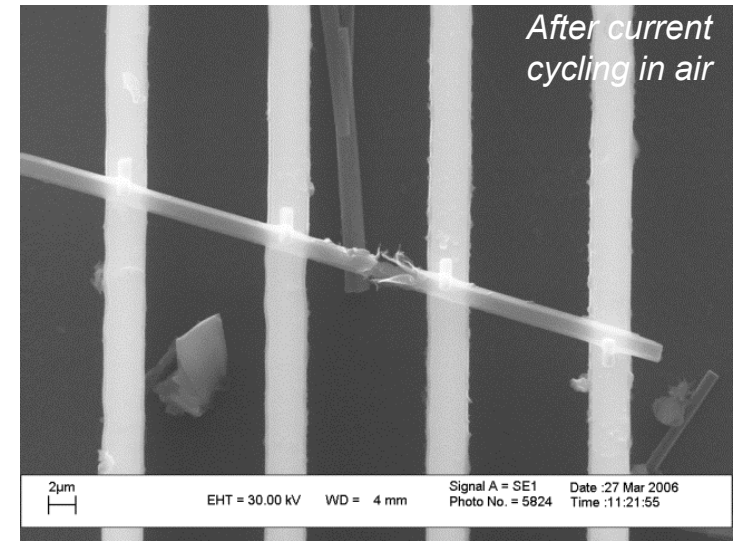

b)

Figure 8. $\quad \mathrm{C}_{60}$ whisker a) before and b) after electrical characterisation in air.

Figure 9a shows the effect of repeated current sweeps, at increasingly higher currents, on the I-V characteristics of the $650 \mathrm{~nm}$ diameter $\mathrm{C}_{60}$ nanowhisker. The increase in sample resistance, with each sweep, is indicative of progressive structural damage. The sample could tolerate several passes of current, and a maximum power of $0.85 \mu \mathrm{W}$, before fracturing between each of the four measurement electrodes (Figure 9b).

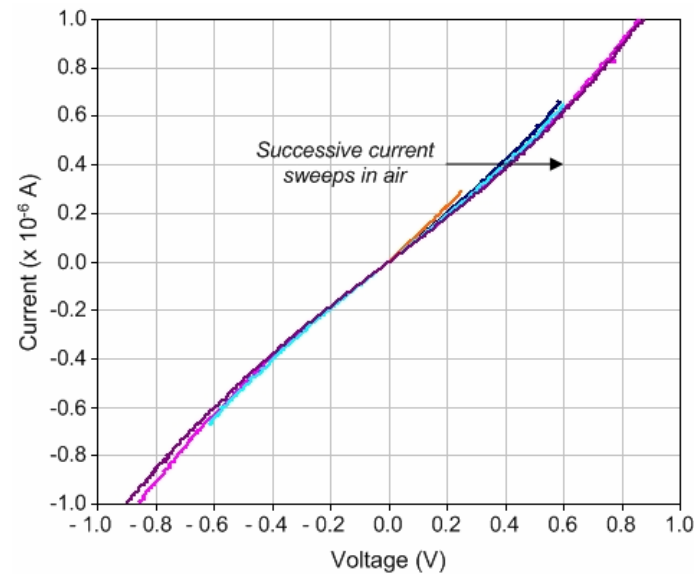

a)

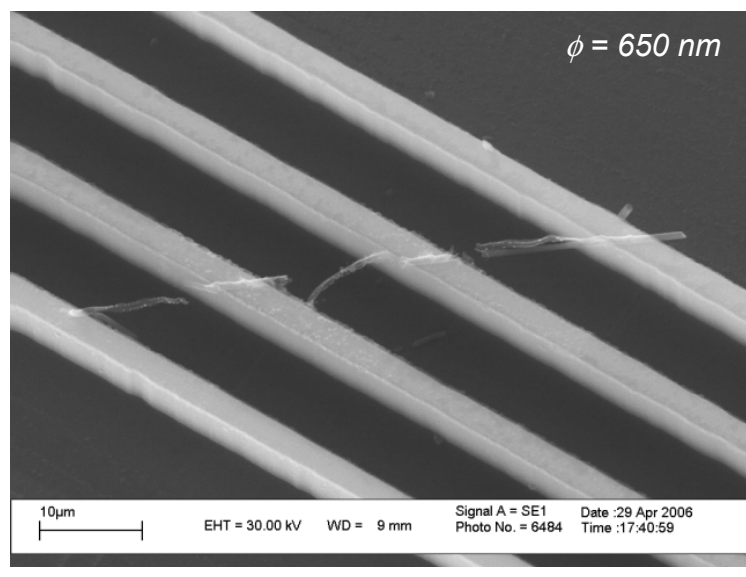

b)

Figure 9. Pogressive damage of a $\mathrm{C}_{60}$ nanowhisker with current: a) increasing resistivity with successive current sweeps; and b) sample after final sweep to $1 \mu \mathrm{A}$. 
Prior to failure, a promisingly low resistivity of $3 \Omega \mathrm{cm}$ was recorded. This value is lower than that measured for the sample in Figures 4 and 6, possibly reflecting the reduction in FIB-processing. Also, as measurements were performed using a 4PP, they exclude contributions due to contact and track resistances. Contact resistances at sampletrack and pad-(stainless steel) probe interfaces will dominate the overall additional contribution to measured resistivity in Figure $6 a$.

Degradation of the $\mathrm{C}_{60}$ whisker structure may hinder their successful use in electronic applications. This problem is not limited to $\mathrm{C}_{60}$ whiskers, however, as a number of studies have reported similar current-induced degradation in single- and multi-walled CNTs in air and under vacuum $[11,12]$. In the case of multi-walled CNTs, failure initiated at a certain power, and progressed as sharp drops in current-carrying capacity, believed to correspond to the concentric destruction of individual graphene walls. The initiation of failure is accelerated in air, however, the overall failure rate is observed to be faster under vacuum. The gradual rise in electrical resistivity for the $\mathrm{C}_{60}$ nanowhisker in Figure 9 can thus be attributed to the gradual destruction of the structure, causing a reduction in currentcarrying capacity. Closer inspection of the images in Figures 6, 8 and 9 suggest degradation initiates in outer layers of both $\mathrm{C}_{60}$ whisker and nanowhisker structures, gradually progressing to inner regions as the voltage drop (and power) across the sample increases. Weaker intermolecular bonding in outer regions of the structures, or a higher availability of oxygen to permit oxidation, are possible reasons for the observed mode of failure. As with CNTs, current-induced degradation may only occur once a certain power threshold is exceeded. Further work is, therefore, needed to investigate this possibility. If true, $\mathrm{C}_{60}$ nanowhiskers could still be used in oxidising environments for low-power electronic applications. An example may be in high-sensitivity, RF power detectors [7], which reduce the detectable signal level by a few orders of magnitude compared to conventional devices, based on Schottky diodes.

\section{Contacting electrodes}

Measurements could only be obtained from $\mathrm{C}_{60}$ whiskers having diameters in excess of $5 \mu \mathrm{m}$, and the high impedance of such samples limited currents to the pico-ampere range. These currents were below the required threshold to enable 4PP measurements to be made with the $\mu$-4PP system, meaning that only $2 \mathrm{PP}$ data could be obtained. Figure 10 shows $\mathrm{I}-\mathrm{V}$ data collected from a $10 \mu \mathrm{m}$ diameter $\mathrm{C}_{60}$ whisker, under $\mathrm{N}_{2}$ gas, along a length of approximately $4 \mu \mathrm{m}$.

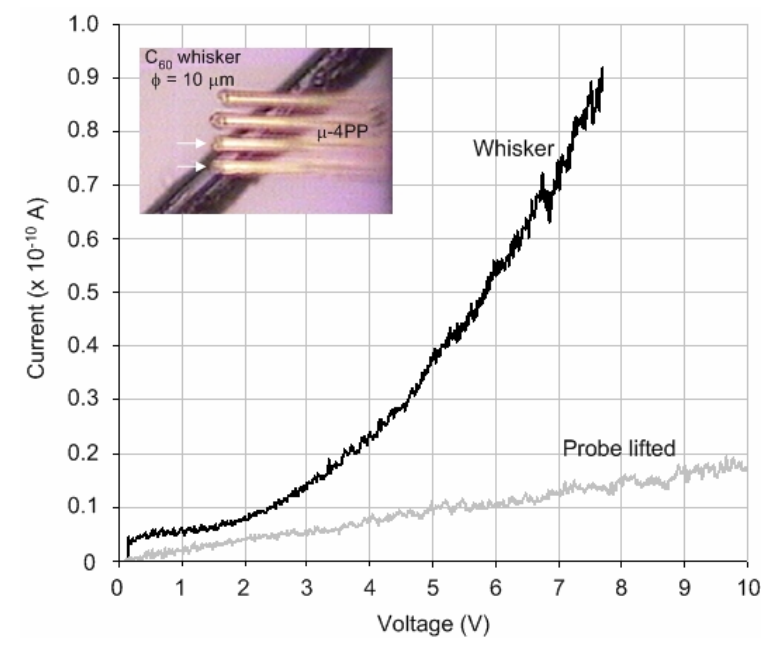

Figure 10. I-V characteristics from $2 \mathrm{PP}$ measurements on a $\mathrm{C}_{60}$ whisker of diameter $10 \mu \mathrm{m}$. Inset: $\mu-4 \mathrm{PP}$ probe during measurement (active electrodes indicated with arrows). 
Data was extracted using the southern-most electrodes on the probe (inset, Figure 10), yielding a sample resistivity of $2.3 \times 10^{8} \Omega \mathrm{cm}$. The true resistivity is likely to be considerably lower than this, however, as the result includes significant contributions due to contact resistance between the whisker and the active electrodes. Successive sweeps of current did not give rise to observable sample degradation. This could be because measurements were conducted in a nitrogen atmosphere, or because sample currents (hence temperatures) were too low to induce the levels of thermal strain necessary to damage the structure.

DC resistivity data gathered from Figures 6, 7 and 10 are included in Figure 2, to augment existing data in the literature. Filled black dots (with intersecting line) represent $4 \mathrm{PP}$ data from the $\mathrm{C}_{60}$ whiskers (and one nanowhisker) attached to planar and raised prepatterned electrodes. The grey dot represents the lowest resistivity measured across a section of the $400 \mathrm{~nm} \mathrm{C}_{60}$ nanowhisker contacted using post-deposited Pt electrodes. Finally, the clear dot indicates data gathered from the $10 \mu \mathrm{m}$ diameter $\mathrm{C}_{60}$ whisker using the contacting $\mu$-4PP. Despite the inherent limitations of each data set, the points are within close agreement with the best-fit curve through measured data obtained in [6].

\section{Conclusions}

Results of $\mathrm{DC}$ characterisation on $\mathrm{C}_{60}$ nanowhiskers are presented for the first time. $\mathrm{A}$ low resistivity of $3 \Omega \mathrm{cm}$ is measured, using a $4 \mathrm{PP}$, on a $650 \mathrm{~nm}$ diameter sample, attached to raised electrodes via FIB-deposited W. By pre-patterning measurement electrodes, the level of FIB-processing can be significantly reduced, minimising sample damage, and improving the quality of the results. The removal of regions of substrate between the electrodes may help to improve data repeatability by eliminating surface-charge interactions with the sample. Current-induced degradation was observed in all samples measured using deposited electrodes, possibly enhanced by oxidation of carbon within the structure. Such degradation may only occur above a certain threshold power level, and further investigation is needed to evaluate this possibility. $\mathrm{C}_{60}$ nanowhiskers may thus still find application in low-power organic electronic applications without the need for a protective inert atmosphere.

A micromachined contacting probe was used in an attempt to eliminate the invasiveness involved in the formation of electrical contacts with $\mathrm{C}_{60}$ whiskers via FIBdeposition of metal. The probe unfortunately proved more harmful to the narrowest whiskers, inducing fracture upon application of pressure, limiting characterisation to whiskers of larger diameter. Work is in progress to modify the $\mu$-4PP system to allow 4PP data to be extracted at lower currents, and trials are planned to obtain data from narrower $\mathrm{C}_{60}$ whiskers using a new system, currently under development. The aim is to try to obtain 4PP data to provide objective comparison with measurements obtained using FIBdeposited metal contacts.

\section{Acknowledgments}

The authors are extremely grateful to Dr. K. Fobelets for useful discussions and Dr. P. Bøggild and Bo Svarrer Hansen for allowing the use of $\mu-4 \mathrm{PP}$ systems at MIC and Capres A/S, respectively. 


\section{References}

[1] H.W. Kroto, J.R. Heath, S.C. O'Brien, R.F. Curl and R.E. Smalley, "C 60 : Buckminsterfullerene", Nature, 318 (6042), pp. 162-163, 1985.

[2] S. Iijima, "Helical microtubules of graphitic carbon", Nature, 354, pp. 56-58, 1991.

[3] M. Nunez-Regueiro, L. Marques, J-L. Hodeau, O. Bethoux and M. Perroux, "Polymerized fullerite structures", Physical Review Letters, 74, 2, pp. 278-281, 1995.

[4] A.M. Rao, P. Zhou, K-A. Wang, G.T. Hager, J.M. Holden, Y. Wang, W-T. Lee, X-X. Bi, P.C. Eklund, D.S. Cornett, M.A. Duncan and I.J. Amster, "Photoinduced polymerization of solid C 60 films", Science, 259, pp. 955-957, 1993.

[5] K. Miyazawa, Y. Kuwasaki, A. Obayashi and M. Kuwabara, " $\mathrm{C}_{60}$ nanowhiskers formed by the liquid-liquid interfacial precipitation method", Journal of Materials Research, Materials Research Society, vol. 17 (1), pp. 83-88, Jan. 2002.

[6] K. Miyazawa, Y. Kuwasaki, K. Hamamoto, S. Nagata, A. Obayashi and M. Kuwabara "Structural characterization of $\mathrm{C}_{60}$ nanowhiskers formed by the liquid/liquid interfacial precipitation method", Surface and Interface Analysis, 35, pp. 117-120, 2003.

[7] S. Lucyszyn, M.P. Larsson, K. Miyazawa, J.-J. Tsaur and M. Ryutaro, “C ${ }_{60}$ AT's Nanowhisker for Quiet Millimetre-Wave Detectors", Invited Paper, CAS International Semiconductor Conference (IEEE), October 3-5, Sinaia, Romania, 2005.

[8] K. Ogawa, T. Kato, A. Ikegami, H. Tsuji and J.P. Bird, "Electrical properties of field-effect transistors based on $\mathrm{C}_{60}$ nanowhiskers", Applied Physics Letters, 88, 112109, 2006.

[9] R. Moret, P. Launois, T. Wagberg and B. Sundqvist, "High-pressure synthesis, structural and Raman studies of a two-dimensional polymer crystal of $\mathrm{C}_{60}$ ", European Physical Journal B, 15, pp. 253-263, 2000.

[10] C.L. Petersen, T.M. Hansen, P. Bøggild, A. Boisen, O. Hansen, T. Hassenkam and F. Grey, "Scanning microscopic four-point conductivity probes", Sensors and Actuators A, 96, pp. 53-58, 2002.

[11] K.A. Dean and B.R. Chalamala, "The environmental stability of field emission from single-walled carbon nanotubes", Applied Physics Letters, Vol. 75, No. 19, pp. 3017-3019, 1999.

[12] P.G. Collins, M. Hersam, M. Arnold, R. Martel and Ph Avouris, "Current saturation and electrical breakdown in multiwalled carbon nanotubes", Vol. 86, No. 14, Physical Review Letters, pp. 3128-3131, 2001. 